\title{
Circulating ESM-1 levels are correlated with the presence of coronary artery disease in patients with obstructive sleep apnea
}

\author{
Haili Sun ${ }^{1,2}$, Fang Fang ${ }^{1,2}$, Kun Li ${ }^{1}$, Huina Zhang ${ }^{2}$, Ming Zhang ${ }^{3}$, Lichuan Zhang ${ }^{1}$, Juan Li ${ }^{2}$, Yanwen Qin ${ }^{2,3}$ and \\ Yongxiang $\mathrm{Wei}^{1,2^{*}}$
}

\begin{abstract}
Background: Endothelial dysfunction is one of the most important early indicators of atherosclerosis in obstructive sleep apnea (OSA) patients. Endothelial cell specific molecules-1 (ESM-1), which is a novel endothelial dysfunction marker that may be linked to cardiovascular disease. We investigated to assess whether circulating ESM-1 levels are correlated with the presence of coronary artery disease (CAD) in patients with OSA.

Methods: We performed a cross-sectional study in 228 Chinese OSA subjects, including 185 patients with OSA and 43 controls. The Gensini stenosis scoring system was used to assess the severity of CAD. Circulating ESM-1 levels were measured by Human Magnetic Luminex Screening Assay. The associations between circulating ESM-1 levels and CAD were determined by multivariate logistic regression analysis. The association between ESM-1 levels and Gensini scores was determined by multivariate linear regression analysis.

Results: CAD patients had significantly higher circulating ESM-1 levels compared with non-CAD patients $(1279.01[918.52-1770.71] \mathrm{pg} / \mathrm{ml}$ vs $585.46[423.61-812.56] \mathrm{pg} / \mathrm{ml}, P<0.001)$. After adjusting for confounding factors, we found that circulating ESM-1 levels were an independent risk factor for CAD $(\mathrm{OR}=1.633 / 100 \mathrm{pg} E S M-1,95 \% \mathrm{Cl}=$ 1.179-2.262, $P=0.003$ ), while circulating ESM-1 levels have no significant correlation with the Gensini score. Furthermore, circulating ESM-1 showed higher discriminatory accuracy in predicting the presence of OSA (AUC: 0.910).
\end{abstract}

Conclusions: Circulating ESM-1 might function as a useful biomarker for monitoring the development and progression of CAD in OSA patients.

Keywords: Obstructive sleep apnea, Endothelial cell specific molecules-1(ESM-1), Coronary artery disease, Biomarker, Endothelial dysfunction

\section{Background}

Obstructive sleep apnea (OSA) is a common sleep-related breathing disorder caused by recurrent collapse of the upper airway during sleep leading to intermittent airway obstruction and absence of airflow despite respiratory efforts of the diaphragm against the occluded pharynx. These respiratory disturbances are associated

\footnotetext{
*Correspondence: weiyongxiang2018@163.com

'Department of Otolaryngology, Beijing Anzhen Hospital, Capital Medical University, NO.2 Anzhen Road, Beijing 100029, China

${ }^{2}$ Key Laboratory of Upper Airway Dysfunction-related Cardiovascular

Diseases, Beijing Institute of Heart, Lung and Blood Vessel Diseases, Beijing

Anzhen Hospital, No. 2 Anzhen Road, Beijing 100029, China

Full list of author information is available at the end of the article
}

with an increased risk for cardiovascular disease, including coronary artery disease, hypertension, and stokes, atherosclerosis [1-4]. The cardiovascular co-morbidities of OSA are assessed by a variety of techniques including measurement of blood pressure, electrocardiogram, exercise testing, echocardiography, and if needed coronary angiography. However, in contrast to the field of cardiovascular diseases, there is currently no established role for blood biomarkers in the diagnosis and risk stratification of cardiovascular consequences/co-morbidities in patients with OSA [5]. Noninvasive blood biomarkers have increasingly emerged as important alternatives to

(C) The Author(s). 2019 Open Access This article is distributed under the terms of the Creative Commons Attribution 4.0 International License (http://creativecommons.org/licenses/by/4.0/), which permits unrestricted use, distribution, and 
traditional methods for diagnosing and stratifying the risk of disease at its earliest stage.

ESM-1, previously named endothelial cell-specific molecule-1, is a new biomarker of endothelial dysfunction [6]. Lassalle et al found that ESM-1 was highly expressed in atheromatous plaques, speculating that the increase in secretion of ESM-1 may promote the migration and proliferation of vascular smooth muscle cells and ESM-1 may play a key role in the pathology of atherosclerosis [7]. Previous study have verified that ESM-1 might play a key role in endothelial dysfunction by promoting adhesion between monocytes and endothelial cells in inflammatory disorders [8]. It has been reported that blood levels of ESM-1 are elevated in patients with coronary artery disease $(\mathrm{CAD})$ or OSA $[6,9,10]$. However, there is no study that investigated circulating ESM-1 levels and cardiovascular risk of CAD in OSA patients, the relationship between circulating ESM-1 levels and the development and progression of $\mathrm{CAD}$ in patients with OSA has never been fully elucidated. However, the relationship between circulating ESM-1 levels and the development and progression of CAD in patients with OSA has never been fully elucidated. Therefore, the present study aims to investigate the correlation of circulating ESM-1 levels with the presence and severity of CAD in patients with OSA.

\section{Materials and methods Study design}

This study was cross-sectional study done in the sleep center (Beijing Anzhen Hospital, China). The study was approved by the ethics committee of Beijing Anzhen Hospital (appoval NO:2017005). All patients gave written informed consent to the ethically approved protocols.

All consecutive patients who underwent overnight full polysomnography study due to snoring, apnea, and excessive daytime sleepiness in the sleep center of Beijing Anzhen Hospital between March 2017 and March 2018 were included in this study. A total of 476 patients were eligible for the study. Subjects younger than 18 years, with central sleep apnea syndrome, heart failure, arrhythmias, stroke, chronic kidney disease, chronic obstructive pulmonary diseases (COPD), pulmonary hypertension, active infections and malignancy were excluded from the study. CAD was defined as present if any of the following characteristics was observed: history of physician-diagnosed CAD, use of medications for CAD; the presence of $\geq 50 \%$ luminal stenosis in at least one major coronary artery in coronary angiography [11]. While if patients have significant cardiac syndromes and not performed coronary angiography before, conventional invasive coronary angiography were performed according to standard protocols by interventional cardiologists blinded to the study protocol. If patients have history of physician-diagnosed CAD or use of medications for CAD were also diagnosed as CAD, no cardiac angiography be performed. All patients gave their written informed consent to participate in the study.

A final total of 228 participants were consecutively enrolled, including 185 patients with OSA and 43 healthy controls (Additional file 1: Figure 1). All the OSA patients were classified into patients with and without CAD. According to the diagnostic standard, the OSA patients were divided into two groups: non-CAD $(n=117)$ and CAD $(n=68)$.

Data on patient characteristics, including clinical/ biochemical factors, were collected. Body mass index (BMI) was calculated by dividing weight in kilograms by squared height in meters $\left(\mathrm{kg} / \mathrm{m}^{2}\right)$. Blood pressure (BP) was measured at the nondominant arm in the morning of the procedure after a $5 \mathrm{~min}$ resting interval. Sleepiness was evaluated using the Epworth sleepiness scale (ESS) [12]. We also recorded the medication status of all the participants (Additional file 2: Table S1).

\section{Coronary angiography analysis}

Conventional invasive coronary angiography was performed according to standard protocols by interventional cardiologists blinded to the study protocol. The Gensini stenosis scoring system was used to assess the severity of CAD [13] by two independent experienced observers. Gensini system grades stenosis of the lumen as 1 for $1-25 \%$ stenosis, 2 for $26-50 \%$ stenosis, 4 for $51-75 \%$ stenosis, 8 for $76-90 \%$ stenosis, 16 for $91-99 \%$ stenosis and 32 for total occlusion. This score was then multiplied by a factor that accounted for the importance of a lesion's position in the coronary arterial tree. The multiplication factor for a left main stem lesion was 5 . The multiplication factor was 2.5 for proximal left anterior descending artery (LAD) and proximal circumflex artery lesions, 1.5 for a mid-LAD lesion, and 1 for distal LAD, mid/distal circumflex artery, and right coronary artery lesions. The multiplication factor for any other branch was 0.5 . The severity of disease was expressed as the sum of the scores for the individual lesions [14].

\section{Laboratory tests}

All blood samples were collected after the participants had fasted overnight. Blood samples were then centrifuged for $10 \mathrm{~min}$ at $3000 \mathrm{rpm}$ and $4{ }^{\circ} \mathrm{C}$. Plasma samples were subsequently stored in a freezer at $-80^{\circ} \mathrm{C}$ before analysis. Serum triglyceride (TG), total cholesterol (TC), low-density lipoprotein cholesterol (LDL-C), high density lipoprotein cholesterol (HDL-C) levels and fasting blood glucose (FBG), and other routine serum biochemical parameters were measured in a biochemical analyzer (Hitachi-7600, Tokyo, Japan) using blinded quality control 
specimens in the Department of the Biochemical Laboratory at Beijing Anzhen Hospital.

Magnetic Luminex ${ }^{\circ}$ Assay is a magnetic bead-based antibody microarray founded upon the sandwich immunoassay principle, which can be used to assess the levels of multiple biomarkers with high sensitivity $[15,16]$. Plasm ESM-1 levels were quantitated by using a Human Magnetic Luminex Screening Assay (R\&D Systems, Minneapolis, MN, USA). The concentrations of ESM-1 in plasm were determined according to the manufacturer's instructions [17]. In order to determine the precision of the standards and cytokines levels values obtained by the Luminex platforms, coefficient of variation $(\mathrm{CV} \%)$ was used to calculate intraassay performance to determine the precision of results. Intra-assay $\mathrm{CV}<10 \%$ was acceptable [18]. In our study, the intra-assay $\mathrm{CV}$ of standard was $<4.0 \%$. Acquisition was performed on the Bio-Plex system (Bio-Rad Laboratories) with the Bio-Plex 3D reader (Luminex FlexMAP 3D) in combination with xPONENT software version 4.2 (Luminex). Data were analyzed by 5 -parameter curve fitting using Bio-Plex Manager software, version 6.1.1 (Bio-Rad).

\section{Statistical analysis}

The study was designed as a cross-sectional study. The sample size was calculated by PASS 11.0 (NCSS, LLC, Kaysville, UT, USA) using logistic regression models, with $P=0.9$, alpha $=0.05, \mathrm{P} 0=0.5$, and odds ratio $(\mathrm{OR})=$ 1.633. The sample size of 228 according to the calculation could satisfy the meet of this study. Continuous variables are expressed as mean \pm standard deviation or median (interquartile range) and categorical variables as numerals (percentages). The independent Student's $t$ test for normal distribution and the Wilcoxon rank sum test for asymmetric distribution were used to analyze the differences in continuous variables. The Chi square test was used to analyze categorical variables. The association between circulating ESM-1 and OSA was determined by multivariate logistic regression analysis. The association between circulating ESM-1 levels and other inflammatory factors were also evaluated using multivariable liner regression analysis. To evaluate the predictive power of the identified predictors of CAD, we used receiver operating characteristic (ROC) curves and in particular the associated area under the curve (AUC). The ROC curves were made with maternal factors alone and combined with different factors to find the best performing model. A $P$ value of $<0.05$ was considered statistically significant. Statistical analysis was performed with SPSS 19.0 (IBM Corp., Armonk, NY, USA).

\section{Results}

Baseline clinical characteristics of the study population The baseline clinical characteristics of the study groups are shown in Table 1 . The present study included 43 controls, $68 \mathrm{CAD}$ patients and 117 non-CAD patients. There were no differences in BMI $(P=0.184)$, TG $(P=$ $0.132)$, FBG $(P=0.088)$, ALT $(P=0.144)$, AST $(P=0.053)$, SBP $(P=0.115)$ between the two groups. Compared with the non-CAD patients, CAD Patients had a significantly difference in HDL-C levels $(P=0.029)$, ODI $(P<0.001)$, Longest apnea time $(P<0.001), C T 90 \%(P<0.001)$ and arousal index $(P<0.001)$ than those without CAD. As shown in Fig. 1, CAD patients had significantly higher circulating ESM-1 levels compared with non-CAD patients (1279.01[918.52-1770.71] pg/ml vs 585.46[423.61-812.56] $\mathrm{pg} / \mathrm{ml}, P<0.001)$.

\section{Association between circulating ESM-1 levels and CAD}

We used ordinal logistic regression analysis to estimate associations between CAD and clinical or biochemical variables. As shown in Table 2, older age $(\mathrm{OR}=1.091$, $95 \% \mathrm{CI}=1.056-1.126, P<0.001), \quad \mathrm{LSaO}_{2} \quad(\mathrm{OR}=1.114$, $95 \% \mathrm{CI}=1.065-1.116, P<0.001)$, and ESM-1 levels $(\mathrm{OR}=$ $1.258,95 \% \mathrm{CI}=1.150-1.376, P<0.001)$ were risk factors for CAD. However, HDL-C levels were a protective factor for $\mathrm{CAD}(\mathrm{OR}=0.900,95 \% \mathrm{CI}=0.654-1.239, P=$ $0.520)$.

The association between CAD and circulating ESM-1 levels were tested in different models of logistic regression. Patients with CAD who had higher circulating ESM-1 levels had a higher OR $(\mathrm{OR}=1.383 / 100 \mathrm{pg}$ ESM$1,95 \% \mathrm{CI}=1.247-1.534, P<0.001$, Table 3$)$. After adjustment for conventional CAD risk factors, including age, sex, BMI, smoking and drinking history, DBP, TG, TC, LDL-C, FBG, ALT and AST, increased ESM-1 levels showed a higher OR of CAD (OR $=1.688 / 100$ pg ESM-1, 95\% CI $=1.226-2.323, P<0.001)$. We also adjusted AHI and $\mathrm{LSaO}_{2}$, we found that elevated circulating ESM-1 levels were associated with higher risk of CAD (OR $=$ $1.633 / 100$ pg ESM-1, 95\% CI =1.179-2.262, $P=0.003$ ).

\section{ROC curve analysis for CAD and ESM-1}

ROC curve analysis suggested that the optimum ESM-1 level cut-off point for patients with CAD was $964.46 \mathrm{pg} /$ $\mathrm{ml}$, with a corresponding sensitivity and specificity of 81.2 and $90.6 \%$, respectively $(\mathrm{AUC}=0.910,95 \% \mathrm{CI}=$ 0.846-0.937, $P<0.001$ ) (Fig. 2).

\section{Association between circulating ESM-1 levels and Gensini score}

Spearmans' correlation was used to examine the association of circulating ESM-1 levels and the Gensini score. Circulating ESM-1 levels have no positive correlation with the Gensini score (rho $=0.079, P=0.534$, Table 4), which represents the severity of CAD in this study. 
Table 1 Baseline clinical characteristics of the study population

\begin{tabular}{|c|c|c|c|c|}
\hline \multirow[t]{2}{*}{ Variables } & \multirow{2}{*}{$\begin{array}{l}\text { Controls } \\
(n=43)\end{array}$} & \multicolumn{3}{|l|}{ OSA } \\
\hline & & non-CAD $(n=117)$ & $\mathrm{CAD}(n=68)$ & $P$ value \\
\hline Male, $n(\%)$ & $29(67.44 \%)$ & 97 (82.91\%) & $61(89.70 \%)$ & 0.207 \\
\hline Ages (years) & $48.4 \pm 16.1$ & $45.6 \pm 11.7$ & $56.3 \pm 9.9$ & $<0.001^{* *}$ \\
\hline Smoke, $n(\%)$ & $14(32.56 \%)$ & $39(33.30 \%)$ & $47(69.12 \%)$ & $<0.001^{* *}$ \\
\hline Alcohol, $n(\%)$ & $6(13.93 \%)$ & $28(23.93 \%)$ & $39(57.35 \%)$ & $<0.001^{* *}$ \\
\hline $\mathrm{BMI}\left(\mathrm{kg} / \mathrm{m}^{2}\right)$ & $23.77 \pm 3.78$ & $27.83 \pm 4.51$ & $27.06 \pm 3.27$ & 0.184 \\
\hline $\mathrm{SBP}(\mathrm{mmHg})$ & $120(110-129)$ & $128(120-136)$ & $123(118-132.5)$ & 0.115 \\
\hline $\mathrm{DBP}(\mathrm{mmHg})$ & $74(69-80)$ & $81(74-90)$ & $76.5(70-89)$ & $0.001^{*}$ \\
\hline $\mathrm{TC}(\mathrm{mmol} / \mathrm{l})$ & $4.73 \pm 1.06$ & $5.09(4.40-5.95)$ & $4.16(3.47-4.95)$ & $<0.001^{* *}$ \\
\hline $\mathrm{TG}(\mathrm{mmol} / \mathrm{l})$ & $1.47(0.99-2.16)$ & $1.54(1.11-2.10)$ & $1.40(1.06-1.73)$ & 0.132 \\
\hline HDL-C (mmol/l) & $1.23(1.08-1.50)$ & $1.20(1.00-1.38)$ & $1.06(0.87-1.20)$ & $0.029^{*}$ \\
\hline LDL-C (mmol/l) & $2.72 \pm 1.08$ & $3.27 \pm 0.96$ & $2.53 \pm 0.88$ & $<0.001^{* *}$ \\
\hline $\mathrm{FBG}(\mathrm{mmol} / \mathrm{l})$ & $5.13 \pm 1.30$ & $5.60 \pm 1.63$ & $5.94 \pm 1.64$ & 0.088 \\
\hline $\mathrm{ALT}(\mathrm{mmol} / \mathrm{l})$ & $20(13-27)$ & $24.50(16-37)$ & $27(22-37)$ & 0.144 \\
\hline AST (mmol/l) & $20.49 \pm 4.98$ & $24.39 \pm 12.26$ & $27.26 \pm 12.42$ & 0.053 \\
\hline Creatinine $(\mathrm{mmol} / \mathrm{l})$ & $61(54.1-74.6)$ & $70.65(60.2-79.05)$ & $67.55(60.28-79.08)$ & 0.656 \\
\hline Uric acid (mmol/l) & $331.30 \pm 79.64$ & $408.27 \pm 100.13$ & $370.34 \pm 90.33$ & $0.012^{*}$ \\
\hline Homocysteine (umol/l) & $9.9(8.5-15.0)$ & $11.55(9.10-15.05)$ & $11.8(9.45-16.10)$ & 0.547 \\
\hline CRP (mg/l) & $0.82(0.27-2.12)$ & $1.58(0.75-2.97)$ & $1.32(0.5-3.95)$ & 0.389 \\
\hline ESM-1 $(p g / m l)$ & $304.24(114.48-628.32)$ & $585.46(423.61-812.56)$ & $1279.01(918.52-1770.71)$ & $<0.001^{* *}$ \\
\hline $\mathrm{AHI}$ & $2.80(1.6-3.70)$ & $37.5(20.5-60.4)$ & $31.05(21.88-45.70)$ & $<0.001^{* *}$ \\
\hline $\mathrm{LSaO}_{2}(\%)$ & $92(91-93)$ & $80(68.5-86)$ & $85(75.5-88)$ & $<0.001^{* *}$ \\
\hline $\mathrm{MSaO}_{2(\%)}$ & $97(95.8-97.07)$ & $96(95.2-97)$ & 93 (93-95) & 0.062 \\
\hline ODI & $1.50(0-2.65)$ & $9.3(2.38-91.8)$ & $36.1(26.35-54.25)$ & $<0.001^{* *}$ \\
\hline Longest apnea time(s) & $23.00(0-39.00)$ & $36.2(24.5-76.75)$ & $76(41.5-107)$ & $<0.001^{* *}$ \\
\hline $\operatorname{MAD}(s)$ & $24(18.75-27)$ & $27(19-35.9)$ & $27(22.95-31.85)$ & $<0.001^{* *}$ \\
\hline СТ90(\%) & $0(0-0.10)$ & $5.8(0.5-26.5)$ & $14.5(0.8-34.3)$ & $<0.001^{* *}$ \\
\hline Arl & $10.65(4.58-15.8)$ & $29.9(18.3-46.5)$ & $35.6(22.4-48.5)$ & $<0.001^{* *}$ \\
\hline ESS & $7(4-10)$ & $12(8-15)$ & $8(4-11)$ & $0.003^{*}$ \\
\hline cIMT (mm) & $0.95 \pm 0.21$ & $1.17 \pm 0.27$ & $1.32 \pm 0.34$ & $0.023^{*}$ \\
\hline
\end{tabular}

Results are expressed as mean \pm standard deviation, median (interquartile range) or $\mathrm{n}(\%)$. Differences between non-CAD group and CAD group were analyzed by the independent Student $t$ test, $x^{2}$ text, or Wilcoxon test

$B M I$ Body mass index, SBP Systolic blood pressure, DBP Diastolic blood pressure, TC Total cholesterol, TG Triglyceride, HDL-C High density lipoprotein, $L D L-C$ Low density lipoprotein, FBG Fasting blood glucose, ALT Alanine aminotransferase, AST Aspartate aminotransferase, CRP C reactive protein, AHI Apnea-hypopnea index, $\mathrm{LSaO}_{2}$ Lowest oxygen saturation, $\mathrm{MSaO}_{2}$ Mean oxygen saturation, ODI Oxygen desaturation index, MAD Mean apnea-hypopnea duration, CT90 Percentage of cumulative time with oxygen saturation below 90\%, Arl Arousal index, ESS Epworth sleepiness scale

$* P<0.05, * * P<0.001$

\section{Discussion}

In this study, we found that circulating ESM-1 levels of CAD patients with OSA were significantly elevated compared with non-CAD patients. Circulating ESM-1 levels were positively associated with the severity of CAD after adjusting for confounding factors. Circulating ESM-1 levels were an independent risk factor for CAD. To the best of our knowledge, the present study is the first addressing the relationship between ESM-1 levels and CAD in OSA patients. ESM-1is a marker for vascular pathology and a mediator of inflammation and adhesion, strongly associated with cardiovascular disease [19]. It is secreted upon stimulation by cytokines, namely tumor necrosis factor- $\alpha$ (TNF- $\alpha$ ), interleukin (IL)-1 and microbial lipopolysaccharide, as well as by proangiogenic factors such as vascular endothelial growth factor (VEGF) [20]. Via its interaction with intercellular adhesion molecules, ESM-1 exhibits a well-described inhibitory role on leukocyte binding to the vascular endothelium. These properties have highlighted its potential role as a biomarker of endothelial dysfunction and 


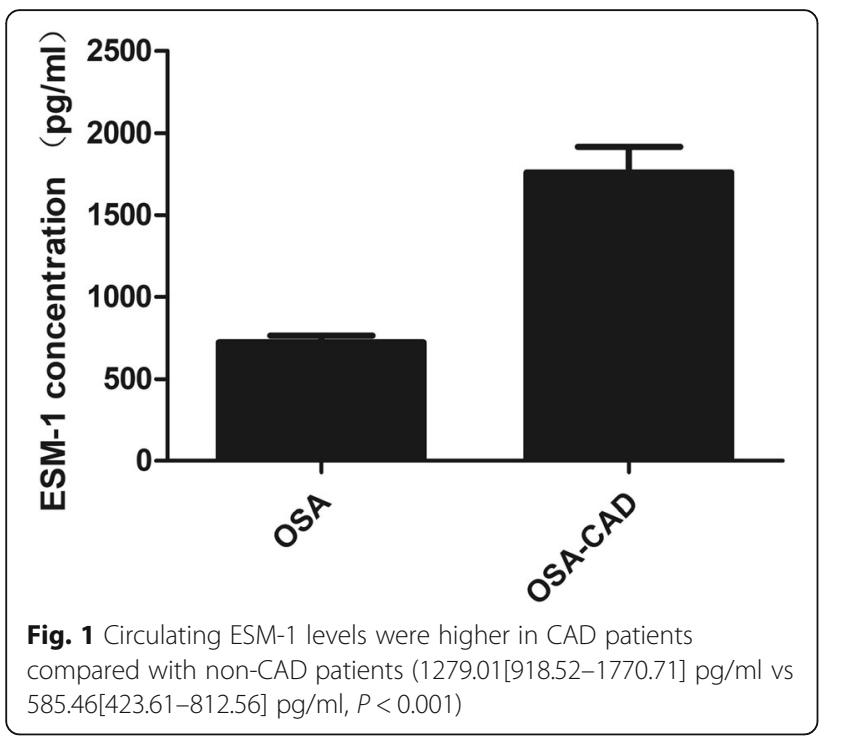

inflammation. In conclusion, since ESM-1 release probably reflects vascular damage and risk factors, its measurement may provide useful information for the management of patients with OSA.

Endothelial dysfunction is considered as the initial lesion in the progress of early atherosclerosis [21]. OSA is one of the most important factors in the pathogenesis of

Table 2 Association between clinical or biochemical variables and CAD

\begin{tabular}{|c|c|c|c|}
\hline Variables & OR & $95 \% \mathrm{Cl}$ & $P$ value \\
\hline Age (year) & 1.091 & $1.056-1.126$ & $<0.001^{* *}$ \\
\hline Gender $($ male $=1$, female $=2$ ) & 1.906 & $0.765-4.753$ & 0.166 \\
\hline $\mathrm{BMI}\left(\mathrm{kg} / \mathrm{m}^{2}\right)$ & 0.954 & $0.884-1.029$ & 0.220 \\
\hline $\mathrm{SBP}(\mathrm{mmHg})$ & 0.991 & $0.972-1.014$ & 0.379 \\
\hline $\mathrm{DBP}(\mathrm{mmHg}$ & 0.962 & $0.934-0.991$ & $0.012^{*}$ \\
\hline $\mathrm{TC}(\mathrm{mmol} / \mathrm{l})$ & 0.397 & $0.278-0.568$ & $<0.001^{* *}$ \\
\hline $\mathrm{TG}(\mathrm{mmol} / \mathrm{l})$ & 0.723 & $0.514-1.016$ & 0.062 \\
\hline $\mathrm{HDL}-\mathrm{C}(\mathrm{mmol} / \mathrm{l})$ & 0.900 & $0.654-1.239$ & 0.520 \\
\hline LDL-C (mmol/l) & 0.405 & $0.273-0.599$ & $<0.001^{* *}$ \\
\hline FBG (mmol/l) & 1.134 & $0.942-1.366$ & 0.185 \\
\hline $\mathrm{ALT}(\mathrm{mmol} / \mathrm{l})$ & 1.002 & $0.988-1.016$ & 0.817 \\
\hline AST (mmol/l) & 1.017 & $0.990-1.045$ & 0.207 \\
\hline AHI (events/h) & 0.966 & $0.951-0.981$ & $<0.001^{* *}$ \\
\hline $\mathrm{LSaO}_{2}(\%)$ & 1.114 & $1.065-1.116$ & $<0.001^{* *}$ \\
\hline ESM-1(per100pg) & 1.258 & $1.150-1.376$ & $<0.001^{* *}$ \\
\hline
\end{tabular}

Dependent variable: $C A D$

$B M I$ Body mass index, SBP Systolic blood pressure, DBP Diastolic blood pressure, TC Total cholesterol, TG Triglyceride, HDL-C High density lipoprotein, $L D L-C$ Low density lipoprotein, FBG Fasting blood glucose, ALT Alanine aminotransferase, AST Aspartate aminotransferase, AHI Apnea-hypopnea index, $\mathrm{LSaO}_{2}$ Lowest oxygen saturation, ESM-1 Endothelial cell specific molecules-1 ${ }^{*} P<0.05$ in ordinal logistic regression analysis, ${ }^{* *} P<0.001$ in ordinal logistic regression analysis
CAD, ESM-1 may play an important role in regulating cell adhesion and raised circulating levels may reflect endothelial dysfunction. Tadzic [22] et al confirmed that the decrease of ESM-1 could reduce the activation of endothelial cells, thus delaying the progress of atherosclerosis. Kose et al. demonstrated that plasma ESM-1 concentrations correlated positively with both markers of inflammation, and ESM-1 levels were significantly increasing in patients with acute coronary syndrome (ACS) compared with the control group. There was no relationship between the Gensini and SYNTAX score and ESM-1 levels; however, there was a significant relationship between ESM-1 levels and the number of diseased epicardial vessels, predicting circulating ESM-1 were associated with the burden of CAD instead of severity of CAD [19].

OSA is associated with an increased risk of cardiovascular disease. Endothelial dysfunction is widely regarded as being involved in the development of atherosclerosis. Carotid artery intima-media thickness (cIMT) is a marker of subclinical atherosclerosis [23]. This result is in accordance with previous studies by Wang et al and Balta et al which revealed that serum ESM-1 levels correlated positively with cIMT in patients with hypertension and psoriasis [24, 25]. Gensini stenosis score is a valid and reliable scoring system that assesses the extent severity of CAD [14]. One of the important findings of the present study was that there was no significant correlation between serum ESM-1 levels, Gensini and SYNTAX score [24]. Kanbay A et al found that ESM-1 levels were significantly higher and flow-mediated dilatation (FMD) measurements were lower in patients with OSA compared to healthy controls and observed a strong negative correlation between serum ESM-1 level and FMD [6]. In our study, we also discuss the relationship of cIMT between these two groups, and found that cIMT were lower in patients with OSA compared to CAD groups. ESM-1 may be a surrogate endothelial dysfunction marker and may have a functional role in endothelium-dependent pathological disorders. All these results suggested that elevated circulating ESM-1 levels might be also correlated with the progression of CAD in OSA patients [19, 25].

Our study showed that circulating ESM-1 levels were positively correlated with age, BMI, SBP, DBP (Additional file 3: Table S2), which can be explained that our study is a cross-sectional study, CAD often occurs when OSA progresses to a certain extent. Epidemiological evidence indicates that blood lipid levels increase with increasing age [26]. However, in our study consistent lower LDL and TC in CAD patients may be the effect of statins or lipid lowering drugs, we found no significant difference in the expression levels of ESM-1 between patients with oral lipid- 


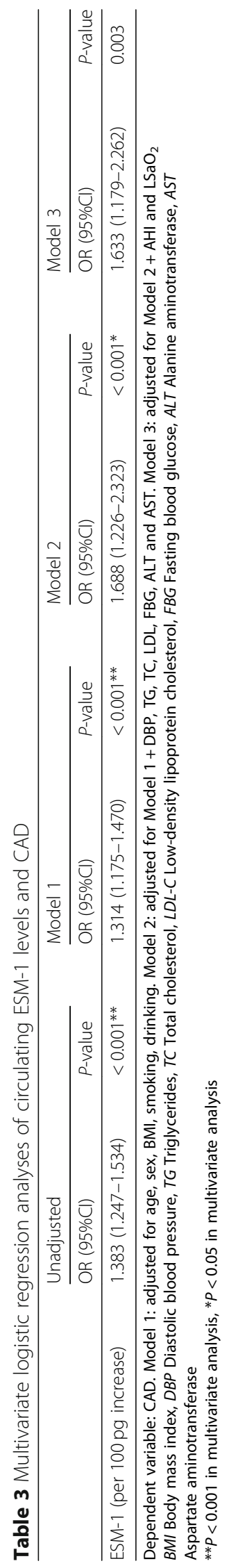




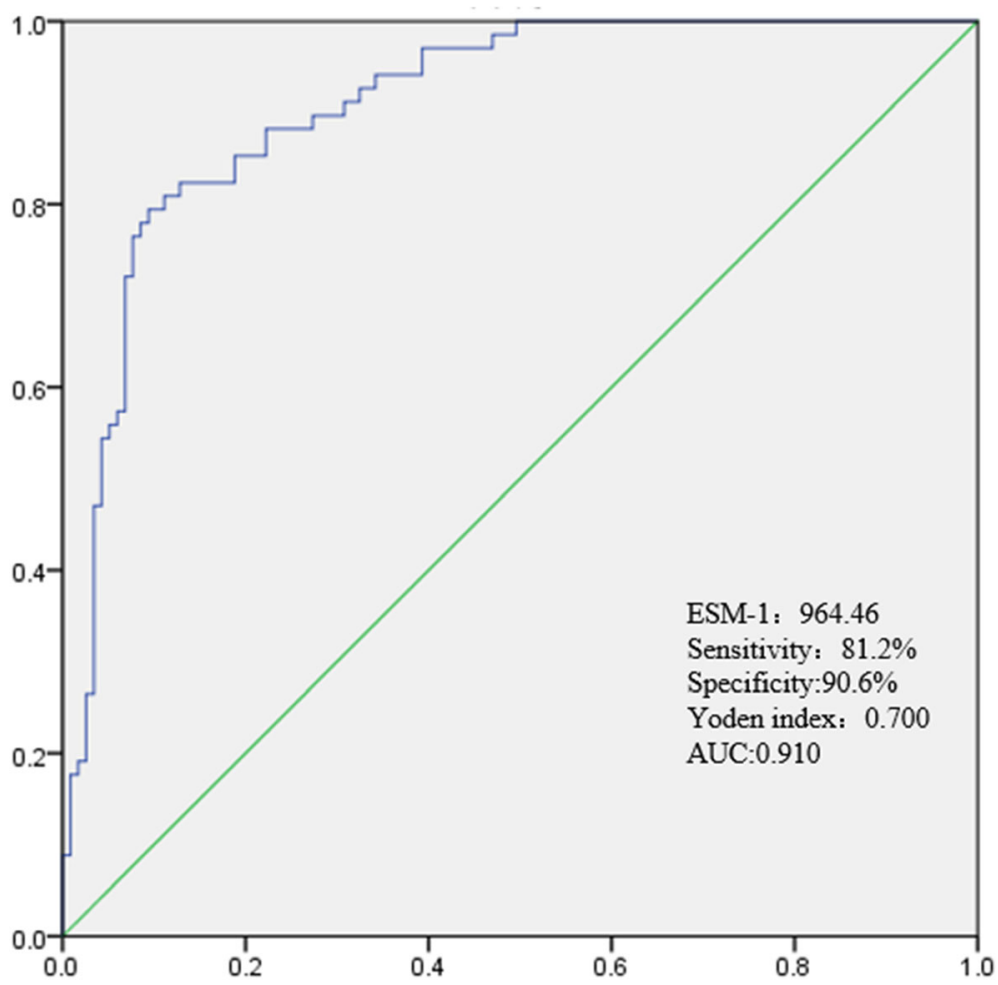

Fig. 2 Receiver-operating characteristic (ROC) curve of ESM-1 for predicting CAD. AUC, area under the curve

lowering drugs and those without oral lipid-lowering drugs. In this study, we found that there were no differences in the levels of circulating ESM-1 between the two genders. There is a higher prevalence of smoking and drinking in patients with CAD and OSA. In order to avoid a confounding effect, we also adjusted for sex, smoking and drinking in this study. A previous study showed that BMI are positively associated with ESM-1 levels [6]. While there were no differences in the levels of ALT and AST in CAD compared with non-CAD in our study. In order to avoid a confounding effect, we also adjusted for BMI, ALT and AST in this study. Hypertension, diabetes and renal function is independently associated with circulating ESM-1 levels and ESM-1 levels are closely correlated with blood pressure, FBG, serum uric acid

Table 4 Correlations between Gensini score and ESM-1 level, $\mathrm{AHI}$ and $\mathrm{LSaO}_{2}$

\begin{tabular}{lll}
\hline Variables & rho & $P$ value \\
\hline ESM-1 & 0.079 & 0.534 \\
$\mathrm{AHI}$ & -0.210 & 0.295 \\
$\mathrm{LSaO}_{2}$ & 0.064 & 0.617
\end{tabular}

ESM-1 Endothelial cell specific molecules-1; $\mathrm{AHI}$ Apnea-hypopnea index, $\mathrm{LSaO}_{2}$ Lowest oxygen saturation

${ }^{*} P<0.05$ in Spearmans' correlation analysis, ${ }^{* *} P<0.001$ in Spearmans correlation analysis and creatinine levels [26-28]. We adjusted for blood pressure, FBG, serum uric acid and creatinine levels in this study.

Care was taken to avoid bias in our study. Human Magnetic Luminex Screening Assay was performed according to the manufacturer's instructions by a trained experimenter who was unaware of patients' clinical data. Moreover, in statistical analysis, adjustments were made for the confounding effects of risk factors for CAD and circulating ESM-1 levels. Finally, propensity score matching was used to reduce the effects of outcome-selection bias. This study has some limitations. First, it was a cross-sectional study, which indicated that it could only show associations, not causality. Second, some of the patients with OSA and some controls were taking drugs, which may affect circulating ESM-1 levels in this study. Third, all of the participants were Chinese, the findings may not be generalizable to other ethnicities. Our findings should be confirmed in other populations.

ESM-1 is a candidate to become a reliable inflammatory indicator of endothelial dysfunction developing before the presentation of the apparent cardiovascular diseases. Therefore, ESM-1 has the potential to shape the therapies regarding the primary prevention of the cardiovascular diseases. Further studies are needed to verify this propose. 


\section{Conclusions}

In summary, we revealed that circulating ESM-1 levels were independently correlated with the presence of CAD in patients with OSA. These findings indicated that ESM-1 might function as a useful biomarker for monitoring the development and progression of CAD in OSA patients. The efficacy of therapies to target ESM-1 to delay the degenerative process of CAD warrants further investigations.

\section{Additional files}

Additional file 1: Figure S1. Flow chart of inclusion cases and controls in this study. (TIF $463 \mathrm{~kb}$ )

Additional file 2: Table S1. List of medications for participants. (DOCX $15 \mathrm{~kb})$

Additional file 3: Table S2. Correlations between clinical variables and circulating ESM-1 levels. (DOCX $16 \mathrm{~kb}$ )

\section{Abbreviations}

CAD: Coronary artery disease; ESM-1: Endothelial cell specific molecules-1; OSA: Obstructive sleep apnea

\section{Acknowledgements}

We are grateful to all of the patients for agreeing to take part in our study. This work was supported by National Natural Science Foundation of China (81870355); Beijing Municipal Administration of Hospitals Clinical Medicine Development of Special Funding Support (ZYLX201605);

Beijing Natural Science Foundation (7192031).

\section{Authors' contributions}

All the authors have accepted responsibility for the entire content of this submitted manuscript and approved submission.

\section{Funding}

We are grateful to all of the patients for agreeing to take part in our study. This work was supported by National Natural Science Foundation of China (81870355); Beijing Municipal Administration of Hospitals Clinical Medicine Development of Special Funding Support (ZYLX201605); Beijing Medical Project (2016-4).

\section{Availability of data and materials}

All the data from this manuscript is publically available.

\section{Ethics approval and consent to participate}

This study was cross-sectional study done in the sleep center (Beijing Anzhen Hospital, China). The study was approved by the ethics committee of Beijing Anzhen Hospital (appoval NO:2017005). All patients gave written informed consent to the ethically approved protocols.

\section{Consent for publication}

All the authors have accepted responsibility for the entire content of this submitted manuscript and approved submission.

\section{Competing interests}

The funding organization(s) played no role in the study design; in the collection, analysis, and interpretation of data; in the writing of the report; or in the decision to submit the report for publication.

\section{Author details}

'Department of Otolaryngology, Beijing Anzhen Hospital, Capital Medical University, NO.2 Anzhen Road, Beijing 100029, China. ${ }^{2}$ Key Laboratory of Upper Airway Dysfunction-related Cardiovascular Diseases, Beijing Institute of Heart, Lung and Blood Vessel Diseases, Beijing Anzhen Hospital, No. 2 Anzhen Road, Beijing 100029, China. ${ }^{3}$ Department of Cardiology, Beijing
Anzhen Hospital, Capital Medical University, NO.2 Anzhen Road, Beijing 100029, China.

Received: 6 April 2019 Accepted: 24 July 2019

Published online: 20 August 2019

\section{References}

1. Muxfeldt ES, Margallo VS, Guimarães GM, Salles GF. Prevalence and associated factors of obstructive sleep apnea in patients with resistant hypertension. Am J Hypertens. 2014;27:1069-78.

2. Shah NA, Yaggi HK, Concato J, Mohsenin V. Obstructive sleep apnea as a risk factor for coronary events or cardiovascular death. Sleep Breath. 2010; 14:131-6.

3. Yaggi HK, Concato J, Kernan WN, Lichtman JH, Brass LM, et al. Obstructive sleep apnea as a risk factor for stroke and death. N Engl J Med. 2005;353: 2034-41.

4. Arnaud C, Dematteis M, Pepin JL, Baguet JP, Lévy P. Obstructive sleep apnea, immuno-inflammation, and atherosclerosis. Semin Immunopathol. 2009;31:113-25.

5. Maeder MT, Mueller C, Schoch OD, Ammann P, Rickli H. Biomarkers of cardiovascular stress in obstructive sleep apnea. Clin Chim Acta. 2016;460: 152-63.

6. Kanbay A, Ceylan E, Köseoğlu HI, Çalışkan M, Takir M, Tulu S, et al. Endocan: a novel predictor of endothelial dysfunction in obstructive sleep apnea syndrome. Clin Respir J. 2018;12:84-90.

7. Lassalle P, Molet S, Janin A, Heyden JV, Tavernier J, Fiers W, et al. ESM-1 is a novel human endothelial cell-specific molecule expressed in lung and regulated by cytokines. J Biol Chem. 1996;271:20458-64.

8. Sun HL, Zhang HN, Li K, Wu H, Zhan XJ, Fang F, et al. ESM-1 promotes adhesion between monocytes and endothelial cells under intermittent hypoxia. J Cell Physiol. 2019;234:1512-21.

9. Çimen T, Efe TH, Akyel A, et al. Human endothelial cell-specific Molecule-1 (Endocan) and coronary artery disease and microvascular angina. Angiology. 2016;67:846-53

10. Xiong C, Zhao ZW, Chen ZY, et al. Elevated human endothelial cell-specific molecule-1 level and its association with coronary artery disease in patients with hypertension. J Investig Med. 2015:63(7):867-70.

11. Lundberg AK, Jonasson L, Hansson GK, Rkw M. Activation-induced FOXP3 isoform profile in peripheral CD4+ T cells is associated with coronary artery disease. Atherosclerosis. 2017;267:27-33.

12. Johns MW. A new method for measuring daytime sleepiness: the Epworth sleepiness scale. Sleep. 1991;14:540-5.

13. Cui G, Li Z, Li R, Huang J, Wang H, Zhang L, et al. A functional variant in APOA5/A4/C3/A1 gene cluster contributes to elevated triglycerides and severity of CAD by interfering with microRNA 3201 binding efficiency. J Am Coll Cardiol. 2014:64:267-77.

14. Gensini GG. A more meaningful scoring system for determining the severity of coronary heart disease. Am J Cardiol. 1983:51:606.

15. Cui L, Shu C, Liu Z, Tong W, Cui M, Wei C, et al. Serum protein marker panel for predicting preeclampsia. Pregnancy Hypertens. 2018;14:279-85.

16. Testlmans D, Tamisier R, Barone-Rochette G, Baguet JP, Roux-Lombard P, Pepin $J$, et al. Profile of circulating cytokines: impact of OSA, obesity and acute cardiovascular events. Cytokine. 2013;62:210-6.

17. Wen WW, Ning $Y$, Zhang $Q$, Yang YX, Jia YF, Sun HL, et al. TNFRSF11B: a potential plasma biomarker for diagnosis of obstructive sleep apnea. Clin Chim Acta. 2019;490:39-45

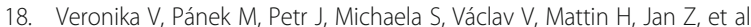
Endothelial microvesicles and soluble markers of endothelial injury in critically ill newborns. Mediat Inflamm. 2018:2018:1-8.

19. Kose M, Emet S, Akpinar TS, Kocaaga M, Cakmak R, Akarsu M, et al. Serum Endocan level and the severity of coronary artery disease: a pilot study. Angiology. 2015;66:727-31.

20. Kali A, Shetty KS. Endocan: a novel circulating proteoglycan. Indian J Pharm. 2014:46:579-83.

21. Balta I, Balta S, Koryurek OM, Demirkol S, Celik T, Akbay G, et al. Mean platelet volume is associated with aortic arterial stiffness in patients with Behçet's disease without significant cardiovascular involvement. J Eur Acad Dermatol Venereol. 2014;28:1388-93.

22. Tadzic R, Mihalj M, Vcev A, Ennen J, Tadzic A, Drenjancevic I. The effects of arterial blood pressure reduction on endocan and soluble endothelial cell adhesion molecules (CAMs) and CAMs ligands expression in hypertensive 
patients on Ca-channel blocker therapy. Kidney Blood Press Res. 2013;37: 103-15.

23. Balci DD, Balci A, Karazincir S, Ucar E, Lyigun U, Yalcin F, et al. Increased carotid artery intima-media thickness and impaired endothelial function in psoriasis. J Eur Acad Dermatol Venereol. 2009;23:1-6.

24. Wang XS, Yang W, Luo T, Wang JM, Jing YY. Serum endocan levels are correlated with the presence and severity of coronary artery disease in patients with hypertension. Genet Test Mol Biomarkers. 2015;19:124-7.

25. Balta I, Balta S, Demirkol S, Mikhailidis DP, Celik T, Akhan M, et al. Elevated serum levels of endocan in patients with psoriasis vulgaris: correlations with cardiovascular risk and activity of disease. Br J Dermatol. 2013;169:1066-70.

26. Yang W, Xiao J, Yang Z, Ji L, Jia W, Weng J, et al. Serum lipids and lipoproteins in Chinese men and women. Circulation. 2012;125:2212-21.

27. Celik T, Balta S, Karaman M, Ahmet AS, Demirkol S, Ozturk C, et al. Endocan, a novel marker of endothelial dysfunction in patients with essential hypertension: comparative effects of amlodipine and valsartan. Blood Press. 2015;24:55-60

28. LV Y, Zhang Y, Shi W, Liu J, Li Y, Zhou Z, et al. The association between Endocan levels and subclinical atherosclerosis in patients with type 2 diabetes mellitus. Am J Med Sci. 2017:353:433-8.

\section{Publisher's Note}

Springer Nature remains neutral with regard to jurisdictional claims in published maps and institutional affiliations.

Ready to submit your research? Choose BMC and benefit from:

- fast, convenient online submission

- thorough peer review by experienced researchers in your field

- rapid publication on acceptance

- support for research data, including large and complex data types

- gold Open Access which fosters wider collaboration and increased citations

- maximum visibility for your research: over $100 \mathrm{M}$ website views per year

At BMC, research is always in progress.

Learn more biomedcentral.com/submissions 